\title{
Factors Associated with Poststroke Fatigue: A Systematic Review
}

\author{
Amélie Ponchel, ${ }^{1,2}$ Stéphanie Bombois, ${ }^{3}$ Régis Bordet, ${ }^{1}$ and Hilde Hénon ${ }^{2}$ \\ ${ }^{1}$ Department of Pharmacology, University of Lille, INSERM U1171, 59045 Lille, France \\ ${ }^{2}$ Department of Neurology, Stroke Center, University of Lille, INSERM U1171, 59045 Lille, France \\ ${ }^{3}$ Department of Neurology, Memory Clinic, University of Lille, INSERM U1171, 59045 Lille, France \\ Correspondence should be addressed to Amélie Ponchel; amelie.ponchel@gmail.com
}

Received 28 February 2015; Revised 20 April 2015; Accepted 12 May 2015

Academic Editor: Alexander C. H. Geurts

Copyright (C) 2015 Amélie Ponchel et al. This is an open access article distributed under the Creative Commons Attribution License, which permits unrestricted use, distribution, and reproduction in any medium, provided the original work is properly cited.

\begin{abstract}
Background. Poststroke fatigue (PSF) is a frequent, disabling symptom that lacks a consensual definition and a standardized evaluation method. The (multiple) causes of PSF have not been formally characterized. Objective. To identify factors associated with PSF. Method. A systematic review of articles referenced in MEDLINE. Only original studies having measured PSF and potentially associated factors were included. Data was extracted from articles using predefined data fields. Results. Although PSF tends to be more frequent in female patients and older patients, sociodemographic factors do not appear to have a major impact. There are strong associations between PSF and emotional disturbances (such as depression and anxiety). PSF may also be linked to attentional disturbances (mainly slowing in processing speed). The literature data have failed to demonstrate a clear impact of the type and severity of stroke. It has been suggested that PSF results from alterations in the frontothalamostriatal system and/or inflammatory processes. Pain, sleep disorders, and prestroke fatigue also appeared to be associated with PSF. Implications. A better understanding of PSF may improve stroke patient care and facilitate the development of effective treatments.
\end{abstract}

\section{Introduction}

Stroke is the second-ranked cause of mortality in the world and a major cause of disability [1]. Whether ischemic or hemorrhagic, stroke can induce physical disabilities [2] and cognitive, psychological, and behavioral impairments [3]. Poststroke depression is of particular interest [4]. For several decades, fatigue was considered to be a symptom of poststroke depression. However, the fact that depression-free patients frequently complain of fatigue has prompted the examination of "poststroke fatigue" (PSF) as a specific syndrome [5].

At present, there is no consensual, clear definition of PSF and this is partly because of the syndrome's complexity. PSF differs from normal fatigue [6-9] that results from overexertion and is relieved by rest [10]. In fact, PSF is a disease state characterized by a chronic, persistent, excessive lack of energy [10-12] with an impact on activities of daily living [13]. PSF is generally defined in subjective terms as an overall state of feeling: "a feeling of early exhaustion, weariness, and aversion to effort" [14]. This type of fatigue has been studied with qualitative approaches such as patient interviews [15] and patient questionnaires like the Fatigue Severity Scale (FSS) [16, 17].

Hence, the wide range of PSF prevalence values found in the literature (from 16\% [18] up to $74 \%$ [19] of patients) may be due to the variety of tools used to measure PSF (based on either unidimensional or multidimensional conceptual frameworks of fatigue) [12], the lack of a consensual definition, and the heterogeneity of stroke patients in terms of age, stroke type and severity, and comorbidities [11].

Besides being frequent, PSF was judged by between $23 \%$ and $59.5 \%$ of stroke patients to be one of their worst symptoms [5, 20-25]. Although fatigue is thought to be less severe and less specific after stroke than in multiple sclerosis, it seems to have similar functional impacts on psychological functioning and professional, social, and familial activities [26]. Furthermore, PSF has a negative impact on subjective feelings of recovery during rehabilitation [27].

PSF is a major cause of "invisible" handicap because levels of awareness of this condition among relatives, work 
colleagues, and even medical staff are low. Two studies have shown that patients receive little information about PSF [7, 28] and do not understand why they feel fatigued [29]. In turn, this leads to difficulties in coping with fatigue and then anxiety, depression, guilt, and a fall in self-esteem [28]. Fatigue may also lead to misunderstanding of the patient's behavior by his/her family or friends; excessive demands may exceed the patient's abilities, maintain anxiety or depression, and lead to withdrawal from certain activities and social life [7].

PSF probably results from complex, poorly understood interactions between biological, psychosocial, and behavioral phenomena. We consider that it is important to try to understand PSF more deeply and thus identify at-risk patients and develop novel treatments. Here, we performed a systematic review of studies of PSF, with a particular focus on associated factors (whether sociodemographic, psychocognitive, or neurophysiological).

\section{Methods}

2.1. Search Strategy. We systematically searched the MEDLINE database via PubMed (up to May 5, 2015) using logical combinations of the keywords "fatigue," "tiredness," or "exhaustion" with "stroke," "transient ischemic attack" (TIA), "intracranial hemorrhage," or "subarachnoid hemorrhage." We did not apply any time or language limitations.

2.2. Eligibility Criteria. We included only original, observational studies of individuals with stroke (whether first or recurrent, ischemic or hemorrhagic). The studies had to assess PSF (using a single question, a case definition or a fatigue scale) and at least one factor associated with PSF (either as a dichotomous or continuous variable).

Studies were excluded if they (i) lacked primary data (i.e., review articles, editorials, or protocol papers), (ii) were case studies, (iii) did not distinguish between data on stroke patients and data on other participants, (iv) did not quantitatively assess PSF or only recorded physical parameters (e.g., electromyography), and (v) contained no data for a valid analysis of putative associations between PSF and other factors.

Our PubMed search identified 1855 individual records. Firstly, all titles and abstracts were screened for eligibility. We identified 1627 articles that did not match our criteria in terms of the study population (i.e., not stroke patients) and the article format (review articles, editorials, protocol papers, and case studies), leaving 228 relevant studies. The reference lists of retrieved articles were checked for other potentially relevant studies, and one other paper was identified as eligible. Hence, 229 articles were assessed for eligibility. Twelve of these articles could not be obtained, and so 217 full texts of potentially eligible publications were retrieved and read. Forty-five papers were excluded because they were not observational studies (19 were interventional trials and 26 were review articles). Analysis of the Methods sections enabled the exclusion of 44 studies because fatigue had not been assessed quantitatively. Twenty-nine papers did not provide data concerning putative associations between PSF and other factors, and one did not distinguish between data on stroke patients and data on other participants. Thus, 98 papers met our inclusion criteria and were included in the review (Figure 1, constructed in accordance with the Preferred Reporting Items for Systematic Reviews and MetaAnalyses (PRISMA) statement [30]).

2.3. Data Extraction. We extracted data on study characteristics (the sample size and the methods used to assess PSF), participants (age, time since stroke, type of stroke, etc.) and outcomes. In particular, we focused on any reported associations between fatigue and the following:

(i) sociodemographic variables (age, gender, ethnicity, educational level, living alone, marital status, social support, independency, employment, etc.),

(ii) psychological factors (depressive symptoms, anxiety, coping style, quality of life, etc.),

(iii) cognitive measures,

(iv) clinical factors (type of stroke, time since stroke, infarct volume, stroke severity, infarct site, stroke etiology, imaging data, vascular risk factors, etc.),

(v) physical factors (walking activity, upper or lower limb function, aerobic fitness, etc.),

(vi) blood laboratory tests,

(vii) prestroke fatigue, sleeping disorders, and sleepiness,

(viii) pain, appetite, and medications.

\section{Results}

Of the included articles, 96 were written in English, one was written in French and one was written in Korean. The included articles had been published between 1999 and 2015 . The median number of included stroke patients was 100 (range: 9 to 3667). Twenty-seven studies assessed patients having suffered ischemic stroke or TIA, 6 assessed patients with hemorrhage, and 65 studied populations of both types of patients. The time since stroke ranged from the acute phase (less than 15 days after stroke) up to 2 years in 69 studies and was over 2 years in 24 studies. The time since stroke was not specified in 5 papers. Eighty-six of the 98 studies used at least one fatigue scale to evaluate fatigue level, with 4 applying a case definition of fatigue. Eleven studies employed a subsection of a health-related quality of life questionnaire to evaluate fatigue or vitality, and one study asked a single question about fatigue.

\section{Sociodemographic Factors}

Forty-six studies tested for associations between gender and PSF but 33 found no significant differences between males and females $[5,21,23,24,29,31-58]$. Twelve showed a predominance of PSF in females [13, 20, 59-68], and only one study demonstrated a predominance of physical fatigue in males [12]. Similarly, 34 out of 44 studies failed to observe any association between PSF and age $[5,13,23,24,29,31-$ $33,35,37,40,42-46,49-58,60,61,63-65,67-69]$, whereas eight demonstrated a positive association $[20,22,34,36,38$, 


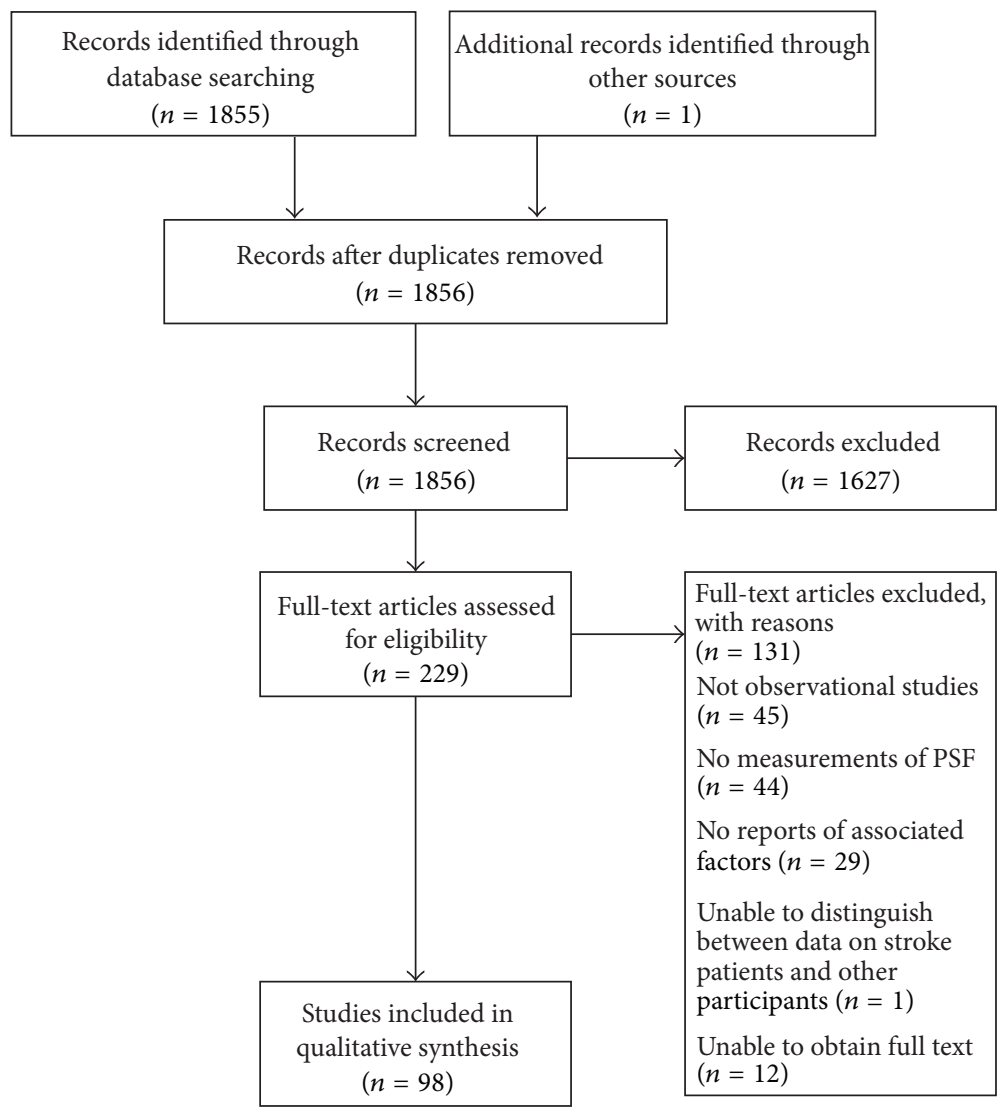

FIgure 1: Study flow diagram.

47, 62, 66], and two demonstrated a negative association $[21,48]$. Educational level (examined in 16 studies) was not associated with PSF $[29,42-44,46,48-52,55,57,64,65,68$, 69]. Ethnicity was not linked to PSF in two studies [32, 42] but was linked in a third study [36].

The impact of social factors on PSF has also been addressed in a few studies but the results remain to be confirmed. Eight studies failed to demonstrate a link between PSF and marital status [21, 23, 42, 43, 47, 55, 58, 64], and only one study found this type of association [62]. The authors of the latter study further postulated that PSF might be more frequent in patients living alone [62]. However, this result was not replicated in four other studies [36, 40, 48, 60]. Lack of social support was associated with more PSF in two studies $[69,70]$ but not in two larger studies $[52,55]$. Lastly, one recent study showed a higher incidence of PSF in patients with dysfunctional familial relationships, patients with a lower family income, and patients living in rural areas [52]. PSF was not associated with family income in one study [69].

Twenty-five studies found that PSF was associated with greater disability and dependency $[20,22,24,25,29,32,33$, $36,38,43-45,48,52-54,57,60,62,64,65,68,71-73]$, even though the association seems to be mediated by depression [74] and was not detected in 15 studies [5, 19, 21, 23, 31, 42, 46, $48-50,56,61,67,69,75]$. PSF was associated with less return to work (and particularly to a return to full-time work) soon or long after the stroke in seven studies $[22,29,43,44,46,76$, 77] but not in another study [64].
Lastly, there are few reported associations between sociodemographic factors and PSF. Other than a trend towards more PSF in females and older patients (as seen for fatigue in the general population [78]), PSF does not seem to be related to educational level, ethnicity, marital status, or social support. Nevertheless, PSF is generally associated with disability, dependency, and infrequent return to work. Thus, PSF has a specific pattern of impact on patients' everyday lives.

\section{Psychocognitive Factors}

5.1. Emotional Disturbances. Fatigue is at least in part a subjective syndrome and depends on psychological factors such as stress linked to stroke itself, having a chronic disease and assuming the consequences of stroke in everyday life [10]. For many years, fatigue was considered to be a symptom of depression because the two conditions were often concomitant [14]; patients with depression are slower and more tired than nondepressed patients [79]. Indeed, patients often confuse fatigue and depression [7].

The great majority of studies (45 out of 48) found a correlation between PSF and poststroke depression [13, 18, 20-22, 24-26, 29, 31, 33, 35-38, 40, 41, 43, 44, 46-50, 52, 53, $57-59,61-65,67-69,72,75,77,80-84]$. Hence, only three studies failed to observe this type of association $[23,55,56]$. The association has been studied at different time points after stroke: in the acute phase [64] and up to 24 months [46]. 
PSF is also associated with suicidality [85]. In the literature, between $29 \%$ and $34 \%$ of fatigued patients are depressed $[5,29,47,74]$. Nonetheless, fatigue was observed in $14-50 \%$ of nondepressed patients [5, 21, 24, 29, 37, 61, 74], and $30 \%$ of fatigued patients did not have any anxious or depressive symptoms [24].

Depression and fatigue might be two separate processes, and the temporal relationship between the two is not well understood [86]. Depression might be a factor in the maintenance of fatigue over time [49]. It is noteworthy that a history of depression (i.e., before stroke) was not always controlled for in these studies. Although one study found that prestroke depression might be linked to PSF [20], another study did not [48].

Although anxiety has been less frequently assessed, it was associated with PSF in 13 studies $[13,22,24,26,33,35,37,44$, $48,57,62,63,65]$ but not in two other studies [23, 55]. This link has been demonstrated from 1 to 18 months after stroke $[22,24,48,63]$.

The style of coping was also associated with the incidence of fatigue: PSF was more frequent in patients with emotionoriented coping [38], passive coping, and external styles of coping $[47,57]$. This result was not confirmed by another study [55].

The negative impact of PSF on quality of life has been demonstrated in 17 studies [19, 21, 32, 33, 40, 69, 77, 86-95].

Emotional factors (such as depression and anxiety) are particularly linked to PSF. At present, the relative influences of these syndromes on each other are not well understood. Nevertheless, we know that there is a clear link between these conditions. Studies of PSF must control for depressive and anxious symptoms as potential confounders. Additionally, PSF is clearly related to quality of life.

5.2. Cognitive Disorders. "Coping theory" states that fatigue is the result of compensatory efforts in response to demands following brain injury [14, 96, 97]. Neuropsychological impairments might contribute to the genesis of fatigue. In this context, the evaluation of cognitive disorders may help us to better understand PSF.

Eleven studies failed to observe correlations between fatigue questionnaire scores and the Mini Mental State Examination (MMSE) [98], even when studying different time points after stroke (from the acute phase to the longer term) $[23,32,43,44,47-49,63,68,75,99]$. One study found that the MMSE score was correlated with PSF but could not predict its change over time [33]. Moreover, this association disappeared when only nondepressed patients were considered, suggesting that cognitive impairment can be mediated by depression [33]. Nevertheless, we know that the MMSE (a measure of general cognitive functioning) is not really sensitive to poststroke attentional and executive disturbances and thus perhaps PSF [100]. The MoCA might provide a more sensitive evaluation of the potential relationships between cognition and PSF [101]. More extensive cognitive batteries might also be more informative.

Selective attention integrates mental, physical, and sensorial inputs when performing a task. Changes in selective attention lead to integration difficulties and thus greater efforts are required to compensate for this impairment [9]. Thus, attentional difficulties might be associated with PSF. Complaints of difficulty in concentrating were found to be associated with PSF up to one year after stroke [72]. One study found that fatigued and nonfatigued patients differed with regard to (i) sustained attention and alertness at 6 months and (ii) sustained attention, alertness, and divided attention at 12 months [22]. However, attentional performances were not related to PSF long after stroke [65]. Although a recent study demonstrated that fatigued and nonfatigued patients did not differ significantly in terms of reaction time [102], processing speed might be related to cognitive/mental fatigue $[18,103]$, physical fatigue [18], but not general fatigue [18]. Processing speed was correlated with PSF 3 and 6 months after stroke [46] and even up to 10 years after stroke [65].

Few studies evaluated PSF and executive functioning. The results did not show any correlation between fatigue scores and mental flexibility $[46,47,103]$. Although inhibition capabilities were correlated with PSF in nondepressed patients in one study [22], this result was not replicated in two other studies $[46,65]$. Fluencies were also associated with fatigue (mainly cognitive fatigue) $[18,22]$, even though three studies failed to demonstrate a link with general fatigue $[46,65,103]$.

Three studies have established a link between language abilities and PSF. One showed that aphasia was predictive of PSF in patients without prestroke fatigue [29]. It has also been demonstrated that patients with language disorders express more fatigue than patients without language disorders [62]. Nevertheless, when evaluating language with specific cognitive measures, the results depend on the time since stroke; one study found a significant association with language abilities 12 months after stroke but not 6 months after stroke [22]. However, the evaluation of aphasic patients is generally challenging, which explains why the potential links between language impairment and PSF are not well understood.

Few studies evaluated links between memory function and PSF. A recent study demonstrated a significant correlation between PSF 6 months after stroke and memory performance in a 10 -word list-learning task [46]. However, the correlation was not significant 3 and 24 months after stroke. Another study evidenced similar fluctuations as a function of the time since stroke [22]. Immediate recall ability has been found to be correlated with (i) cognitive fatigue for verbal material and (ii) physical fatigue for visual memory [18]. In contrast, PSF was not correlated with delayed recall ability for verbal and visual memory $[18,65]$. The results concerning working memory are also disparate, with two studies demonstrating an association $[18,65]$ and one study not demonstrating an association [103]. Further studies are required.

Other cognitive domains have not been extensively explored (visuoconstructive performances [65], visual neglect [29], orientation [21], and reasoning [46]) but do not appear to be associated with PSF.

PSF does not seem to be associated with general cognitive functioning. Some preliminary results demonstrated an association with attentional difficulties but need to be confirmed. At present, the data on executive functioning, memory, and language are too sparse to enable firm conclusions to be drawn. 


\section{Neurophysiological Factors}

6.1. Neurological Factors. One can legitimately hypothesize that neurological disease factors in general (and strokeassociated parameters in particular) have an impact on PSF. However, few studies have evidenced links between PSF and stroke characteristics.

Ischemic and hemorrhagic strokes appeared to have much the same impact on fatigue in ten studies $[5,13,23$, $32,35,42,47,61-63]$. However, the studies often focused on heterogeneous groups with a high proportion of ischemic stroke patients and thus a low proportion of hemorrhagic stroke patients; this might have influenced the statistical significance of the results. Infarct volume was not of importance in nine studies $[29,31,33,45,46,49,50,67,68]$, and neither was thrombolysis [33]. At least one other previous stroke was significantly associated with PSF in four studies $[21,36,62,65]$ but not in 14 studies [20, 23, 31, 33, 44, 48-50, 55, 61, 63, 67, $68,99]$. Neither the time since stroke $[5,23,25,29,42,44,51$, $55,56,61,65,80]$ nor the stroke etiology $[29,31,33,34]$ was correlated with PSF in 12 and 4 studies, respectively.

According to two studies, fatigue was more frequent in patients with TIA $[27,37]$, suggesting that the presence of a lesion can influence PSF. Six studies found that PSF was associated with stroke severity [21, 22, 27, 29, 33, 38, 52], although the association was not significant in nondepressed patients [33]. Sixteen studies did not find this relationship [5, 20, 31, $35,44,46,48-50,53,54,60,63,65,67,68]$. PSF was linked to long-term mortality rates in three studies $[20,43,66]$.

Although PSF is more frequent after stroke than after TIAs, it appears that stroke characteristics (such as the type, severity, etiology, and infarct volume) are not predictive of PSF.

6.2. Imaging Data. In view of the literature data, one can hypothesize that fatigue is related to poor functional integration within the limbic system and the basal ganglia (associated with alterations in the frontothalamostriatal system) [104].

Stroke side was not linked to PSF in 17 studies $[5,13$, $21-23,35,38,39,42,44-48,52,55,63]$. In contrast, other studies found relationships between PSF and various stroke sites: posterior strokes [63], infratentorial lesions [48], and basilar infarcts [44]. However, most studies did not find any relationship between stroke site and PSF $[13,18,20,22,29$, $31,33,35,57,60,65]$. The lesion site might have a differential impact depending on the type of fatigue, with a trend towards more physical fatigue in patients with subcortical lesions and more cognitive fatigue in patients with cortical lesions [18].

Magnetic resonance imaging studies have investigated PSF. In line with the hypothetical involvement of subcorticofrontal systems in nondepressed patients, lesions located in the basal ganglia and internal capsule were found to be predictive of PSF [67], as were caudate infarcts [68]. PSF might also be linked with profound microbleeds [50].

The impact of white matter lesions has rarely been studied. The few available data suggest that fatigue is more frequent in patients with severe leukoaraiosis on a CT scan [20]. Nonetheless, four MRI studies failed to detect a link between white matter lesions and fatigue [33, 49, 50, 68]. However, a recent study of nondepressed patients demonstrated that white matter hyperintensities were not associated with PSF 3 months after stroke but were predictive of fatigue one year after stroke [49].

In summary, lesion side and site are not clearly associated with PSF. However, recent MRI studies have provided data that suggest the involvement of the subcorticofrontal system in fatigue.

6.3. Physical Deconditioning. Some recent studies have focused on physical deconditioning as a potential explanation for PSF. A fall in muscle strength might lead to an increase in the effort demand and thus greater fatigue. Thus, poor physical functioning might contribute to PSF [41, 64].

PSF was significantly related to walk scores (and particularly the number of steps) in four studies [23, 35, 42, 105] but not in three other studies $[21,56,106]$. PSF was not correlated with walking speed $[56,70,107,108]$ or upper or lower limb functioning [21,23], except in one study [109]. Five studies observed correlations with balance, motor control and aerobic fitness $[42,70,83,110,111]$, although six others did not demonstrate significant differences $[23,47,55,56,70,112]$. However, this kind of measurement (gait, balance, etc.) might be more closely correlated with physical fatigue than with general or mental fatigue [18].

Recently, a study investigated the relationship between motor cortex excitability (measured following transcranial magnetic stimulation) and fatigue in stroke patients with minimal impairments [113]. Patients with high levels of fatigue exhibited higher motor thresholds, and those who perceived high physical efforts displayed low excitability of the inputs that drive motor cortex output. The researchers suggested that PSF might result from a difference between the effort produced by the patient and the actual motor output [113].

Physical deconditioning is a promising hypothesis in terms of rehabilitation: physical reconditioning therapies are based on the idea that maximizing activity and mobility could increase force and endurance and thus reduce PSF [86]. Physical fatigue scales and objective measurements (e.g., electromyography) are better indicators of fatigue related to physical condition but were not the focus of our review.

6.4. Biological Factors. To date, biological factors putatively involved in PSF have received little attention: this might nevertheless constitute a promising field in terms of finding pharmacological treatments.

Although some neuroendocrine hypotheses of PSF have been suggested $[9,104]$, cortisol, adrenocorticotropin hormone and thyroid hormone (T4, TSH) levels were not associated with PSF in one study [22].

Inflammatory hypotheses seem more promising but have not been extensively studied $[9,114]$. In a pilot study, PSF was observed in patients with high levels of C-reactive protein [115]. However, this result was not replicated in a larger study [45]. In contrast, PSF was associated with high levels of interleukin- (IL-) $1 \beta$ [45] and with low levels of IL-9 and the neuroprotective IL- $1 \beta$ antagonist IL-ra [45] in the poststroke 
acute phase, which was not the case with poststroke depression [81]. There was no correlation with other inflammatory agents (such as IL-8, IL-18, growth-related oncogene- $\alpha$, IL2 , IL-4, IL-6, IL-10, IL-12, interferon- $\gamma$, and tumor necrosis factor- $\alpha$ ) [45]. A recent study demonstrated a possible genetic contribution to PSF: particular single nucleotide polymorphisms in genes associated with immune response were possibly associated with susceptibility to or protection against PSF [31]. A proinflammatory response might be responsible for the development of fatigue short-term after stroke, which might then be complicated by psychosocial factors [45].

It has also been postulated that biochemical anomalies (such as vitamin B12 deficiency [116], low tryptophan levels, and high kynurenine levels [117]) might be associated with PSF. PSF was also found to be associated with glycemia [45, $53,54]$, uric acid levels [53], and elevated homocysteine levels [54]. Levels of hemoglobin [45], total cholesterol, triglycerides, high-density lipoprotein, low-density lipoprotein, and fibrinogen were not correlated with PSF [54].

Hence, inflammatory factors might be associated with PSF. However, larger studies are needed to confirm the recently published preliminary results.

6.5. Comorbidities. Frequent comorbidities of stroke might also contribute to PSF [10]. Nevertheless, five questionnairebased studies did not find an association between comorbidities and PSF [23, 29, 36, 47, 60].

6.5.1. Vascular Risk Factors. Although vascular risk factors might conceivably be involved in PSF, the results tend to argue against this hypothesis. Three studies demonstrated that PSF was more frequent in patients with heart disease $[20,43,52]$, whereas eight did not observe any difference $[29,31,36,44,45,53,54,61]$. An association with diabetes was observed in three studies (all by the same research group) $[20,43,44]$ but not in twelve others $[29,31,33,35-37,49,52-$ $54,61,68]$. Anemia was not correlated with PSF in one study [61]. An association with hyperlipidemia was found in one study [68] but not in four others [29, 31, 49, 52]. PSF was related to hypertension in one study [37] but not in fourteen others $[20,29,31,33,35,36,44,45,49,50,53,54,68]$. Smoking was associated with PSF in one study [29] but not in five others [31, 36, 43, 44, 52]. Alcohol consumption was also associated with PSF in one study [43] but not in three others $[29,46,52]$. Three studies found that body mass index was not correlated with PSF $[20,44,52]$. Migraine was correlated with PSF in one study only [44].

6.5.2. Sleep Disorders. Sleep disorders are frequent after stroke, TIA [118-121], and subarachnoid hemorrhage [122] and might even constitute a risk factor for stroke [123, 124]. After a stroke, about $50 \%$ of patients complain of changes in their sleeping habits; they notably report sleeping longer at night and being more drowsy during the day [7, 125]. About $30 \%$ reported sleep disorders up to one year after stroke, with daytime sleepiness, longer sleep latency, and nonrefreshing sleep $[47,75]$. Thus, PSF was associated with sleep disorders (assessed by questionnaires) in nine studies [20, 29, 49, $52,64,69,75,122,126]$ but not in three others $[47,55,61]$.
A correlation between PSF and daytime sleepiness was also observed in four studies $[12,35,125,126]$ but not in two others $[36,56]$.

6.5.3. Prestroke Fatigue. Prestroke fatigue might also be associated with PSF. In a study of 220 stroke patients, $38 \%$ reported prestroke fatigue [29]. This factor was strongly associated with PSF in five studies [29, 33, 52, 64, 99], although $36 \%$ of the patients who did not suffer from preexisting fatigue also complained of PSF [29]. Two studies failed to demonstrate a link between pre- and poststroke fatigue [35, 63].

6.5.4. Pain. Seven studies found a significant link between pain and PSF $[20,49,56,62,69,80,127]$. About $10 \%$ of patients displayed the triad of fatigue, depression, and pain, and about $20 \%$ suffered from fatigue and pain but not depression $[20,80]$. Moreover, pain might be involved in the persistence of fatigue over time [49].

6.5.5. Nutrition and Appetite. Nutrition contributes to PSF, since poor nutritional status was associated with low vitality $[69,128]$. Fatigue has also been linked to a decrease in appetite [29].

6.5.6. Medications. Lastly, medications taken to treat frequent comorbidities can also impact on PSF $[9,10]$. Patients often report that their fatigue is due to medications [7]. Unfortunately, few studies have analyzed this potential influence. Three studies did not show any relationship between medications and PSF $[29,45,46]$. A further study found no association between PSF and beta-blockers or statins [61]. Other studies showed an association between PSF and the use of sedative drugs [52], antidepressants [33, 77, 80], hypnotics $[20,80]$, analgesics [20], and antihypertensive drugs [37].

In summary, PSF does not appear to be associated with vascular risk factors. In contrast, PSF is frequently associated with prestroke fatigue and poststroke sleeping disorders and daytime sleepiness. Pain, nutrition, and medications might also be linked to the presence of PSF, although further investigations are needed.

\section{Conclusion}

PSF is a frequent, disabling health condition that results from the complex interaction between the many factors reviewed here.

Although there is a trend towards a greater incidence of PSF in women and in elderly patients, sociodemographic factors (such as educational level, social support, and marital status) do not seem to be significantly associated with PSF. In contrast, psychological and life factors (such as depression, anxiety, and poor quality of life) are strongly linked to PSF. Even though studies evaluating overall cognitive function did not demonstrate correlations with fatigue scores, more extensive cognitive investigations revealed correlations with attentional performances in general and processing speed in particular.

Neurological factors (such as the type, severity, and etiology of stroke) and infarct volume do not appear to 
be associated with PSF. However, the presence of a lesion might be of importance because stroke patients are more fatigued than patients having suffered a TIA. Recent MRI data have suggested the involvement of the subcorticofrontal network in PSF. Biological data are rarely reported in studies of PSF. The inflammatory hypothesis is promising but must be confirmed in larger studies. Lastly, PSF appears to be independent of vascular risk factors but is associated with sleep disorders, prestroke fatigue, pain, and poor nutrition. The patient's medication might also be linked to the presence of PSF, although data on this subject are lacking.

A better understanding of PSF will enable healthcare workers to recognize this "invisible handicap" more frequently and explain it more clearly to their patients. Furthermore, a better understanding of PSF might facilitate the development of effective treatments strategies aiming at fatigue directly or indirectly through the treatment of associated factors.

\section{Conflict of Interests}

The authors have no conflict of interests to declare with regard to the present work.

\section{Acknowledgment}

The authors thank David Fraser (Biotech Communication SARL) for helpful comments on the paper's English.

\section{References}

[1] G. A. Donnan, M. Fisher, M. Macleod, and S. M. Davis, "Stroke," The Lancet, vol. 371, no. 9624, pp. 1612-1623, 2008.

[2] C. D. A. Wolfe, “The impact of stroke," British Medical Bulletin, vol. 56, no. 2, pp. 275-286, 2000.

[3] I. Kneebone and N. B. Lincoln, "Psychological problems after stroke and their management: state of knowledge," Neuroscience \& Medicine, vol. 3, no. 1, pp. 83-89, 2012.

[4] R. M. Dafer, M. Rao, A. Shareef, and A. Sharma, "Poststroke depression," Topics in Stroke Rehabilitation, vol. 15, no. 1, pp. 1321, 2008.

[5] J. L. Ingles, G. A. Eskes, and S. J. Phillips, "Fatigue after stroke," Archives of Physical Medicine and Rehabilitation, vol. 80, no. 2, pp. 173-178, 1999.

[6] V. L. Barbour and G. E. Mead, "Fatigue after stroke: the patient's perspective," Stroke Research and Treatment, vol. 2012, Article ID 863031, 6 pages, 2012.

[7] N. A. Flinn and J. E. Stube, "Post-stroke fatigue: qualitative study of three focus groups," Occupational Therapy International, vol. 17, no. 2, pp. 81-91, 2010.

[8] M. Kirkevold, D. Christensen, G. Andersen, S. P. Johansen, and I. Harder, "Fatigue after stroke: manifestations and strategies," Disability and Rehabilitation, vol. 34, no. 8, pp. 665-670, 2012.

[9] J. Levine and B. D. Greenwald, "Fatigue in Parkinson disease, stroke, and traumatic brain injury," Physical Medicine and Rehabilitation Clinics of North America, vol. 20, no. 2, pp. 347361, 2009.

[10] M. H. de Groot, S. J. Phillips, and G. A. Eskes, "Fatigue associated with stroke and other neurologic conditions: implications for stroke rehabilitation," Archives of Physical Medicine and Rehabilitation, vol. 84, no. 11, pp. 1714-1720, 2003.

[11] S. Choi-Kwon and J. S. Kim, "Poststroke fatigue: an emerging, critical issue in stroke medicine," International Journal of Stroke, vol. 6, no. 4, pp. 328-336, 2011.

[12] M. Falconer, S. Walsh, and J. A. Harbison, "Estimated prevalence of fatigue following stroke and transient ischemic attack is dependent on terminology used and patient gender," Journal of Stroke and Cerebrovascular Diseases, vol. 19, no. 6, pp. 431434, 2010.

[13] J. Lynch, G. Mead, C. Greig, A. Young, S. Lewis, and M. Sharpe, "Fatigue after stroke: the development and evaluation of a case definition," Journal of Psychosomatic Research, vol. 63, no. 5, pp. 539-544, 2007.

[14] F. Staub and J. Bogousslavsky, "Post-stroke depression or fatigue?” European Neurology, vol. 45, no. 1, pp. 3-5, 2001.

[15] G. Eilertsen, H. Ormstad, and M. Kirkevold, "Experiences of poststroke fatigue: qualitative meta-synthesis," Journal of Advanced Nursing, vol. 69, no. 3, pp. 514-525, 2013.

[16] A. Lerdal, L. N. Bakken, S. E. Kouwenhoven et al., "Poststroke fatigue-a review," Journal of Pain and Symptom Management, vol. 38, no. 6, pp. 928-949, 2009.

[17] L. B. Krupp, N. G. LaRocca, J. Muir-Nash, and A. D. Steinberg, "The fatigue severity scale. Application to patients with multiple sclerosis and systemic lupus erythematosus," Archives of Neurology, vol. 46, no. 10, pp. 1121-1123, 1989.

[18] M. Hubacher, P. Calabrese, C. Bassetti, A. Carota, M. Stöcklin, and I.-K. Penner, "Assessment of post-stroke fatigue: the fatigue scale for motor and cognitive functions," European Neurology, vol. 67, no. 6, pp. 377-384, 2012.

[19] I. G. L. van de Port, G. Kwakkel, V. P. M. Schepers, C. T. I. Heinemans, and E. Lindeman, "Is fatigue an independent factor associated with activities of daily living, instrumental activities of daily living and health-related quality of life in chronic stroke?" Cerebrovascular Diseases, vol. 23, no. 1, pp. 4045, 2007.

[20] H. Naess, L. Lunde, J. Brogger, and U. Waje-Andreassen, "Fatigue among stroke patients on long-term follow-up. the Bergen Stroke Study," Journal of the Neurological Sciences, vol. 312, no. 1-2, pp. 138-141, 2012.

[21] N. E. Parks, G. A. Eskes, G. J. Gubitz, Y. Reidy, C. Christian, and S. J. Phillips, "Fatigue impact scale demonstrates greater fatigue in younger stroke survivors," Canadian Journal of Neurological Sciences, vol. 39, no. 5, pp. 619-625, 2012.

[22] N. Radman, F. Staub, T. Aboulafia-Brakha, A. Berney, J. Bogousslavsky, and J.-M. Annoni, "Poststroke fatigue following minor infarcts: a prospective study," Neurology, vol. 79, no. 14, pp. 14221427, 2012.

[23] H. M. van Eijsden, I. G. L. van de Port, J. M. A. Visser-Meily, and G. Kwakkel, "Poststroke fatigue: who is at risk for an increase in fatigue?" Stroke Research and Treatment, vol. 2012, Article ID 863978, 8 pages, 2012.

[24] V. Vuletić, Z. Lezaić, and S. Morović, "Post-stroke fatigue," Acta clinica Croatica, vol. 50, no. 3, pp. 341-344, 2011.

[25] S. P. van der Werf, H. L. P. van den Broek, H. W. M. Anten, and G. Bleijenberg, "Experience of severe fatigue long after stroke and its relation to depressive symptoms and disease characteristics," European Neurology, vol. 45, no. 1, pp. 28-33, 2001.

[26] S. Gramigna, M. Schluep, F. Staub et al., "Fatigue in neurological disease: different patterns in stroke and multiple sclerosis," Revue Neurologique, vol. 163, no. 3, pp. 341-348, 2007. 
[27] C. Winward, C. Sackley, Z. Metha, and P. M. Rothwell, "A population-based study of the prevalence of fatigue after transient ischemic attack and minor stroke," Stroke, vol. 40, no. 3, pp. 757-761, 2009.

[28] J. H. White, K. R. Gray, P. Magin et al., "Exploring the experience of post-stroke fatigue in community dwelling stroke survivors: a prospective qualitative study," Disability and Rehabilitation, vol. 34, no. 16, pp. 1376-1384, 2012.

[29] S. Choi-Kwon, S. W. Han, S. U. Kwon, and J. S. Kim, "Poststroke fatigue: characteristics and related factors," Cerebrovascular Diseases, vol. 19, no. 2, pp. 84-90, 2005.

[30] D. F. Stroup, J. A. Berlin, S. C. Morton et al., "Meta-analysis of observational studies in epidemiology: a proposal for reporting. Meta-analysis of observational studies in epidemiology (MOOSE) group," The Journal of the American Medical Association, vol. 283, no. 15, pp. 2008-2012, 2000.

[31] K. Becker, R. Kohen, R. Lee et al., "Poststroke fatigue: hints to a biological mechanism," Journal of Stroke and Cerebrovascular Diseases, vol. 24, no. 3, pp. 618-621, 2015.

[32] Z. Butt, J.-S. Lai, D. Rao, A. W. Heinemann, A. Bill, and D. Cella, "Measurement of fatigue in cancer, stroke, and HIV using the Functional Assessment of Chronic Illness Therapy-Fatigue (FACIT-F) scale," Journal of Psychosomatic Research, vol. 74, no. 1, pp. 64-68, 2013.

[33] Y.-K. Chen, J.-F. Qu, W.-M. Xiao et al., "Poststroke fatigue: risk factors and its effect on functional status and health-related quality of life," International Journal of Stroke, 2014.

[34] T. J. Chestnut, "Fatigue in stroke rehabilitation patients: a pilot study," Physiotherapy Research International, vol. 16, no. 3, pp. 151-158, 2010.

[35] F. Duncan, S. J. Lewis, C. A. Greig et al., "Exploratory longitudinal cohort study of associations of fatigue after stroke," Stroke, vol. 46, no. 4, pp. 1052-1058, 2015.

[36] V. L. Feigin, S. Barker-Collo, V. Parag et al., "Prevalence and predictors of 6-month fatigue in patients with ischemic stroke: a population-based stroke incidence study in Auckland, New Zealand, 2002-2003," Stroke, vol. 43, no. 10, pp. 2604-2609, 2012.

[37] J. A. Harbison, S. Walsh, and R. A. Kenny, "Hypertension and daytime hypotension found on ambulatory blood pressure is associated with fatigue following stroke and TIA," QJM, vol. 102, no. 2, pp. 109-115, 2009.

[38] K. Jaracz, L. Mielcarek, and W. Kozubski, "Clinical and psychological correlates of poststroke fatigue. Preliminary results," Neurologia i Neurochirurgia Polska, vol. 41, no. 1, pp. 36-43, 2007.

[39] F. Lamb, J. Anderson, M. Saling, and H. Dewey, "Predictors of subjective cognitive complaint in postacute older adult stroke patients," Archives of Physical Medicine and Rehabilitation, vol. 94, no. 9, pp. 1747-1752, 2013.

[40] A. Lerdal and C. L. Gay, "Fatigue in the acute phase after first stroke predicts poorer physical health 18 months later," Neurology, vol. 81, no. 18, pp. 1581-1587, 2013.

[41] A. Lerdal, K. A. Lee, L. N. Bakken, A. Finset, and H. S. Kim, "The course of fatigue during the first 18 months after first-ever stroke: a longitudinal study," Stroke Research and Treatment, vol. 2012, Article ID 126275, 8 pages, 2012.

[42] K. K. Miller, S. A. Combs, M. Van Puymbroeck et al., "Fatigue and pain: relationships with physical performance and patient beliefs after stroke," Topics in Stroke Rehabilitation, vol. 20, no. 4, pp. 347-355, 2013.
[43] H. Naess and H. Nyland, "Poststroke fatigue and depression are related to mortality in young adults: a cohort study," BMJ Open, vol. 3, no. 3, Article ID 002404, 2013.

[44] H. Naess, H. I. Nyland, L. Thomassen, J. Aarseth, and K.-M. Myhr, "Fatigue at long-term follow-up in young adults with cerebral infarction," Cerebrovascular Diseases, vol. 20, no. 4, pp. 245-250, 2005.

[45] H. Ormstad, H. C. D. Aass, K.-F. Amthor, N. Lund-Sørensen, and L. Sandvik, "Serum cytokine and glucose levels as predictors of poststroke fatigue in acute ischemic stroke patients," Journal of Neurology, vol. 258, no. 4, pp. 670-676, 2011.

[46] R. Pihlaja, J. Uimonen, S. Mustanoja, T. Tatlisumak, and E. Poutiainen, "Post-stroke fatigue is associated with impaired processing speed and memory functions in first-ever stroke patients," Journal of Psychosomatic Research, vol. 77, no. 5, pp. 380-384, 2014.

[47] V. P. Schepers, A. M. Visser-Meily, M. Ketelaar, and E. Lindeman, "Poststroke fatigue: course and its relation to personal and stroke-related factors," Archives of Physical Medicine and Rehabilitation, vol. 87, no. 2, pp. 184-188, 2006.

[48] L. Snaphaan, S. van der Werf, and F.-E. de Leeuw, "Time course and risk factors of post-stroke fatigue: a prospective cohort study, European Journal of Neurology, vol. 18, no. 4, pp. 611-617, 2011.

[49] W. K. Tang, Y. K. Chen, H. J. Liang et al., "Subcortical white matter infarcts predict 1-year outcome of fatigue in stroke," BMC Neurology, vol. 14, article 234, 2014.

[50] W. K. Tang, X. X. Liu, Y. K. Chen et al., "Cerebral microbleeds and fatigue in stroke," European Neurology, vol. 71, no. 5-6, pp. 213-216, 2014.

[51] P. O. Valko, C. L. Bassetti, K. E. Bloch, U. Held, and C. R. Baumann, "Validation of the fatigue severity scale in a Swiss cohort," Sleep, vol. 31, no. 11, pp. 1601-1607, 2008.

[52] S. S. Wang, J. J. Wang, P. X. Wang, and R. Chen, "Determinants of fatigue after first-ever ischemic stroke during acute phase," PLoS ONE, vol. 9, no. 10, Article ID e110037, 2014.

[53] D. Wu, L. Wang, W. Teng, K. Huang, and X. Shang, "Correlation of fatigue during the acute stage of stroke with serum uric acid and glucose levels, depression, and disability," European Neurology, vol. 72, no. 3-4, pp. 223-227, 2014.

[54] D. Wu, L. Wang, W. Teng, K. Huang, and X. Shang, "Correlation of post-stroke fatigue with glucose, homocysteine and functional disability," Acta Neurologica Scandinavica, vol. 131, no. 6, pp. 400-404, 2014.

[55] A. M. Zedlitz, A. J. Visser-Meily, V. P. Schepers, A. C. Geurts, and L. Fasotti, "Patients with severe poststroke fatigue show a psychosocial profile comparable to patients with other chronic disease: implications for diagnosis and treatment," ISRN Neurology, vol. 2011, Article ID 627081, 8 pages, 2011.

[56] C. L. Hoang, J.-Y. Salle, S. Mandigout, J. Hamonet, F. MacianMontoro, and J.-C. Daviet, "Physical factors associated with fatigue after stroke: an exploratory study," Topics in Stroke Rehabilitation, vol. 19, no. 5, pp. 369-376, 2012.

[57] P. E. C. A. Passier, M. W. M. Post, M. J. E. van Zandvoort, G. J. E. Rinkel, E. Lindeman, and J. M. A. Visser-Meily, "Predicting fatigue 1 year after aneurysmal subarachnoid hemorrhage," Journal of Neurology, vol. 258, no. 6, pp. 1091-1097, 2011.

[58] E. K. Stokes, C. O’Connell, and B. Murphy, "An investigation into fatigue post-stroke and its multidimensional nature," Advances in Physiotherapy, vol. 13, no. 1, pp. 2-10, 2011. 
[59] A.-H. Almborg, K. Ulander, A. Thulin, and S. Berg, "Discharged after stroke-important factors for health-related quality of life," Journal of Clinical Nursing, vol. 19, no. 15-16, pp. 2196-2206, 2010.

[60] D. Christensen, S. P. Johnsen, T. Watt, I. Harder, M. Kirkevold, and G. Andersen, "Dimensions of post-stroke fatigue: a twoyear follow-up study," Cerebrovascular Diseases, vol. 26, no. 2, pp. 134-141, 2008.

[61] G. A. Crosby, S. Munshi, A. S. Karat, E. Worthington, and N. B. Lincoln, "Fatigue after stroke: frequency and effect on daily life," Disability and Rehabilitation, vol. 34, no. 8, pp. 633-637, 2012.

[62] E.-L. Glader, B. Stegmayr, and K. Asplund, "Poststroke fatigue: a 2-year follow-up study of stroke patients in Sweden," Stroke, vol. 33, no. 5, pp. 1327-1333, 2002.

[63] M. A. Kutlubaev, S. D. Shenkin, A. J. Farrall et al., "CT and clinical predictors of fatigue at one month after stroke," Cerebrovascular Diseases Extra, vol. 3, no. 1, pp. 26-34, 2013.

[64] A. Lerdal, L. N. Bakken, E. F. Rasmussen et al., "Physical impairment, depressive symptoms and pre-stroke fatigue are related to fatigue in the acute phase after stroke," Disability and Rehabilitation, vol. 33, no. 4, pp. 334-342, 2011.

[65] N. A. M. M. Maaijwee, R. M. Arntz, L. C. A. Rutten-Jacobs et al., "Post-stroke fatigue and its association with poor functional outcome after stroke in young adults," Journal of Neurology, Neurosurgery \& Psychiatry, 2014.

[66] G. E. Mead, C. Graham, P. Dorman et al., "Fatigue after stroke: baseline predictors and influence on survival. analysis of data from UK patients recruited in the international stroke trial," PLoS ONE, vol. 6, no. 3, Article ID e16988, 2011.

[67] W. K. Tang, Y. K. Chen, V. Mok et al., "Acute basal ganglia infarcts in poststroke fatigue: an MRI study," Journal of Neurology, vol. 257, no. 2, pp. 178-182, 2010.

[68] W. K. Tang, H. J. Liang, Y. K. Chen et al., "Poststroke fatigue is associated with caudate infarcts," Journal of the Neurological Sciences, vol. 324, no. 1-2, pp. 131-135, 2013.

[69] M. Suh and S. Choi-Kwon, "Structural equation modeling on quality of life in stroke survivors," Journal of Korean Academy of Nursing, vol. 40, no. 4, pp. 533-541, 2010.

[70] K. M. Michael, J. K. Allen, and R. F. Macko, "Fatigue after stroke: relationship to mobility, fitness, ambulatory activity, social support, and falls efficacy," Rehabilitation Nursing, vol. 31, no. 5, pp. 210-217, 2006.

[71] S. Palmcrantz, L. Widén Holmqvist, and D. K. Sommerfeld, "Young individuals with stroke: a cross sectional study of longterm disability associated with self-rated global health," $B M C$ Neurology, vol. 14, no. 1, article 20, 2014.

[72] K. Koopman, M. Uyttenboogaart, P. C. Vroomen, J. van der Meer, J. de Keyser, and G.-J. Luijckx, "Long-term sequelae after cerebral venous thrombosis in functionally independent patients," Journal of Stroke \& Cerebrovascular Diseases, vol. 18, no. 3, pp. 198-202, 2009.

[73] J. Powell, N. Kitchen, J. Heslin, and R. Greenwood, "Psychosocial outcomes at 18 months after good neurological recovery from aneurysmal subarachoid haemorrhage," Journal of Neurology, Neurosurgery and Psychiatry, vol. 75, no. 8, pp. 1119-1124, 2004.

[74] U. M. Badaru, O. O. Ogwumike, A. F. Adeniyi, and O. O. Olowe, "Variation in functional independence among stroke survivors having fatigue and depression," Neurology Research International, vol. 2013, Article ID 842980, 6 pages, 2013.
[75] J. Y. Park, M. H. Chun, S. H. Kang, J. A. Lee, B. R. Kim, and M. J. Shin, "Functional outcome in poststroke patients with or without fatigue," American Journal of Physical Medicine \& Rehabilitation/Association of Academic Physiatrists, vol. 88, no. 7, pp. 554-558, 2009.

[76] G. Andersen, D. Christensen, M. Kirkevold, and S. P. Johnsen, "Post-stroke fatigue and return to work: a 2-year follow-up," Acta Neurologica Scandinavica, vol. 125, no. 4, pp. 248-253, 2012.

[77] A. Vetkas, T. Lepik, T. Eilat, T. Rätsep, and T. Asser, "Emotional health and quality of life after aneurysmal subarachnoid hemorrhage," Acta Neurochirurgica, vol. 155, no. 6, pp. 1107-1114, 2013.

[78] T. Watt, M. Groenvold, J. B. Bjorner, V. Noerholm, N.-A. Rasmussen, and P. Bech, "Fatigue in the Danish general population. Influence of sociodemographic factors and disease," Journal of Epidemiology and Community Health, vol. 54, no. 11, pp. 827833, 2000.

[79] L. D. M. N. Terroni, R. Fráguas, M. de Lucia et al., "Importance of retardation and fatigue/interest domains for the diagnosis of major depressive episode after stroke: a four months prospective study," Revista Brasileira de Psiquiatria, vol. 31, no. 3, pp. 202207, 2009.

[80] H. Naess, L. Lunde, and J. Brogger, "The triad of pain, fatigue and depression in ischemic stroke patients: the bergen stroke study," Cerebrovascular Diseases, vol. 33, no. 5, pp. 461-465, 2012.

[81] H. Ormstad, H. C. D. Aass, K.-F. Amthor, N. Lund-Sorensen, and L. Sandvik, "Serum levels of cytokines, glucose, and hemoglobin as possible predictors of poststroke depression, and association with poststroke fatigue," International Journal of Neuroscience, vol. 122, no. 11, pp. 682-690, 2012.

[82] O. R. F. Smith, K. C. van den Broek, M. Renkens, and J. Denollet, "Comparison of fatigue levels in patients with stroke and patients with end-stage heart failure: application of the fatigue assessment scale," Journal of the American Geriatrics Society, vol. 56, no. 10, pp. 1915-1919, 2008.

[83] B. Y. Tseng, S. A. Billinger, B. J. Gajewski, and P. M. Kluding, "Exertion fatigue and chronic fatigue are two distinct constructs in people post-stroke," Stroke, vol. 41, no. 12, pp. 2908-2912, 2010.

[84] I. G. L. van de Port, G. Kwakkel, M. Bruin, and E. Lindeman, "Determinants of depression in chronic stroke: a prospective cohort study," Disability and Rehabilitation, vol. 29, no. 5, pp. 353-358, 2007.

[85] W.-K. Tang, J.-Y. Lu, V. Mok, G. S. Ungvari, and K.-S. Wong, "Is fatigue associated with suicidality in stroke?" Archives of Physical Medicine and Rehabilitation, vol. 92, no. 8, pp. 13361338, 2011.

[86] F. Duncan, S. Wu, and G. E. Mead, "Frequency and natural history of fatigue after stroke: a systematic review of longitudinal studies," Journal of Psychosomatic Research, vol. 73, no. 1, pp. 1827, 2012.

[87] I. Muus, D. Christensen, M. Petzold et al., "Responsiveness and sensitivity of the Stroke Specific Quality of Life Scale Danish version," Disability and Rehabilitation, vol. 33, no. 25-26, pp. 2425-2433, 2011.

[88] G. Vincent-Onabajo and A. Adamu, "Impact of poststroke fatigue on health-related quality of life of nigerian stroke survivors," Journal of Stroke, vol. 16, no. 3, pp. 195-201, 2014.

[89] J. M. A. Visser-Meily, M. L. Rhebergen, G. J. E. Rinkel, M. J. van Zandvoort, and M. W. M. Post, "Long-term health-related quality of life after aneurysmal subarachnoid hemorrhage relationship with psychological symptoms and personality characteristics," Stroke, vol. 40, no. 4, pp. 1526-1529, 2009. 
[90] S.-Y. Yang and K. H. Kong, "Level and predictors of participation in patients with stroke undergoing inpatient rehabilitation," Singapore Medical Journal, vol. 54, no. 10, pp. 564-568, 2013.

[91] H. Naess, L. Lunde, and J. Brogger, "The effects of fatigue, pain, and depression on quality of life in ischemic stroke patients: the Bergen Stroke Study," Vascular Health and Risk Management, vol. 8, no. 1, pp. 407-413, 2012.

[92] H. Naess, U. Waje-Andreassen, L. Thomassen, H. Nyland, and K.-M. Myhr, "Health-related quality of life among young adults with ischemic stroke on long-term follow-up," Stroke, vol. 37, no. 5, pp. 1232-1236, 2006.

[93] W. K. Tang, J. Y. Lu, Y. K. Chen, V. C. Mok, G. S. Ungvari, and K. S. Wong, "Is fatigue associated with short-term healthrelated quality of life in stroke?" Archives of Physical Medicine and Rehabilitation, vol. 91, no. 10, pp. 1511-1515, 2010.

[94] I. G. L. van de Port, G. A. M. van den Bos, M. Voorendt, G. Kwakkel, and E. Lindeman, "Identification of risk factors related to perceived unmet demands in patients with chronic stroke," Disability and Rehabilitation, vol. 29, no. 24, pp. 1841-1846, 2007.

[95] P. E. C. A. Passier, J. M. A. A. Visser-Meily, G. J. E. Rinkel, E. Lindeman, and M. W. M. Post, "Life satisfaction and return to work after aneurysmal subarachnoid hemorrhage," Journal of Stroke \& Cerebrovascular Diseases, vol. 20, no. 4, pp. 324-329, 2011.

[96] F. Staub and J. Bogousslavsky, "Fatigue after stroke: a major but neglected issue," Cerebrovascular Diseases, vol. 12, no. 2, pp. 75$81,2001$.

[97] A. H. van Zomeren, W. H. Brouwer, and B. G. Deelman, "Attentional deficits: the riddgles of selectivity, speed, and alertness," in Closed Head Injury: Psychological, Social and Familial Consequences, pp. 74-107, Oxford University Press, Oxford, UK, 1984.

[98] M. F. Folstein, S. E. Folstein, and P. R. McHugh, "Mini-mental state. A practical method for grading the cognitive state of patients for the clinician," Journal of Psychiatric Research, vol. 12, no. 3, pp. 189-198, 1975.

[99] F. Duncan, C. Greig, S. Lewis et al., "Clinically significant fatigue after stroke: a longitudinal cohort study," Journal of Psychosomatic Research, vol. 77, no. 5, pp. 368-373, 2014.

[100] A. Bour, S. Rasquin, A. Boreas, M. Limburg, and F. Verhey, "How predictive is the MMSE for cognitive performance after stroke?" Journal of Neurology, vol. 257, no. 4, pp. 630-637, 2010.

[101] R. Lees, J. Selvarajah, C. Fenton et al., "Test accuracy of cognitive screening tests for diagnosis of dementia and multidomain cognitive impairment in stroke," Stroke, vol. 45, no. 10, pp. 30083018, 2014.

[102] A. Kuppuswamy, E. V. Clark, K. S. Sandhu, J. C. Rothwell, and N. S. Ward, "Post-stroke fatigue: a problem of altered corticomotor control?" Journal of Neurology, Neurosurgery \& Psychiatry, 2015.

[103] B. Johansson and L. Rönnbäck, "Mental fatigue and cognitive impairment after an almost neurological recovered stroke," ISRN Psychiatry, vol. 2012, Article ID 686425, 7 pages, 2012.

[104] A. Chaudhuri and P. O. Behan, "Fatigue and basal ganglia," Journal of the Neurological Sciences, vol. 179, no. 1-2, pp. 34-42, 2000.

[105] C. A. Robinson, A. Shumway-Cook, M. A. Ciol, and D. Kartin, "Participation in community walking following stroke: subjective versus objective measures and the impact of personal factors," Physical Therapy, vol. 91, no. 12, pp. 1865-1876, 2011.

[106] K. Michael and R. F. Macko, "Ambulatory activity intensity profiles, fitness, and fatigue in chronic stroke," Topics in Stroke Rehabilitation, vol. 14, no. 2, pp. 5-12, 2007.
[107] S. E. Lord, L. Rochester, M. Weatherall, K. M. McPherson, and H. K. McNaughton, "The effect of environment and task on gait parameters after stroke: a randomized comparison of measurement conditions," Archives of Physical Medicine and Rehabilitation, vol. 87, no. 7, pp. 967-973, 2006.

[108] I. G. van de Port, G. Kwakkel, and E. Lindeman, "Community ambulation in patients with chronic stroke: how is it related to gait speed?" Journal of Rehabilitation Medicine, vol. 40, no. 1, pp. 23-27, 2008.

[109] S. J. Lewis, A. J. Barugh, C. A. Greig et al., "Is fatigue after stroke associated with physical deconditioning? A cross-sectional study in ambulatory stroke survivors," Archives of Physical Medicine and Rehabilitation, vol. 92, no. 2, pp. 295-298, 2011.

[110] B. Y. Tseng and P. Kluding, "The relationship between fatigue, aerobic fitness, and motor control in people with chronic stroke: a pilot study," Journal of Geriatric Physical Therapy, vol. 32, no. 3, pp. 97-102, 2009.

[111] I. G. L. van de Port, G. Kwakkel, I. van Wijk, and E. Lindeman, "Susceptibility to deterioration of mobility long-term after stroke: a prospective cohort study," Stroke, vol. 37, no. 1, pp. 167171, 2006.

[112] S. Durcan, E. Flavin, and F. Horgan, "Factors associated with community ambulation in chronic stroke," Disability and Rehabilitation, pp. 1-5, 2015.

[113] A. Kuppuswamy, E. V. Clark, I. F. Turner, J. C. Rothwell, and N. S. Ward, "Post-stroke fatigue: a deficit in corticomotor excitability?” Brain, vol. 138, no. 1, pp. 136-148, 2015.

[114] M. A. Kutlubaev, F. H. Duncan, and G. E. Mead, "Biological correlates of post-stroke fatigue: a systematic review," Acta Neurologica Scandinavica, vol. 125, no. 4, pp. 219-227, 2012.

[115] F. McKechnie, S. Lewis, and G. Mead, "A pilot observational study of the association between fatigue after stroke and Creactive protein," Journal of the Royal College of Physicians of Edinburgh, vol. 40, no. 1, pp. 9-12, 2010.

[116] M. Huijts, A. Duits, J. Staals, and R. J. van Oostenbrugge, "Association of vitamin B12 deficiency with fatigue and depression after lacunar stroke," PLoS ONE, vol. 7, no. 1, Article ID e30519, 2012.

[117] H. Ormstad, R. Verkerk, K.-F. Amthor, and L. Sandvik, "Activation of the kynurenine pathway in the acute phase of stroke and its role in fatigue and depression following strok," Journal of Molecular Neuroscience, vol. 54, no. 2, pp. 181-187, 2014.

[118] L. N. Bakken, K. A. Lee, H. S. Kim, A. Finset, and A. Lerdal, "Sleep-wake patterns during the acute phase after first-ever stroke," Stroke Research and Treatment, vol. 2011, Article ID 936298, 7 pages, 2011.

[119] C. Bassetti, M. S. Aldrich, R. D. Chervin, and D. Quint, “Sleep apnea in patients with transient ischemic attack and stroke: a prospective study of 59 patients," Neurology, vol. 47, no. 5, pp. 1167-1173, 1996.

[120] C. L. Bassetti and D. M. Hermann, "Sleep and stroke," Handbook of Clinical Neurology, vol. 99, pp. 1051-1072, 2011.

[121] D. M. Hermann and C. L. Bassetti, "Sleep-related breathing and sleep-wake disturbances in ischemic stroke," Neurology, vol. 73, no. 16, pp. 1313-1322, 2009.

[122] W. J. Schuiling, G. J. E. Rinkel, R. Walchenbach, and A. W. de Weerd, "Disorders of sleep and wake in patients after subarachnoid hemorrhage," Stroke, vol. 36, no. 3, pp. 578-582, 2005.

[123] M. Arzt, T. Young, L. Finn, J. B. Skatrud, and T. D. Bradley, "Association of sleep-disordered breathing and the occurrence 
of stroke," The American Journal of Respiratory and Critical Care Medicine, vol. 172, no. 11, pp. 1447-1451, 2005.

[124] D. J. Capampangan, K. E. Wellik, J. M. Parish et al., "Is obstructive sleep apnea an independent risk factor for stroke? A critically appraised topic," Neurologist, vol. 16, no. 4, pp. 269273, 2010.

[125] A. Sterr, K. Herron, D.-J. Dijk, and J. Ellis, “Time to wakeup: sleep problems and daytime sleepiness in long-term stroke survivors," Brain Injury, vol. 22, no. 7-8, pp. 575-579, 2008.

[126] M. Suh, S. Choi-Kwon, and J. S. Kim, "Sleep disturbances after cerebral infarction: role of depression and fatigue," Journal of Stroke and Cerebrovascular Diseases, vol. 23, no. 7, pp. 19491955, 2014.

[127] H. Naess, L. Lunde, J. Brogger, and U. Waje-Andreassen, "Poststroke pain on long-term follow-up: the Bergen stroke study," Journal of Neurology, vol. 257, no. 9, pp. 1446-1452, 2010.

[128] A. Westergren, "Nutrition and its relation to mealtime preparation, eating, fatigue and mood among stroke survivors after discharge from hospital-a Pilot Study," The Open Nursing Journal, vol. 2, no. 1, pp. 15-20, 2008. 


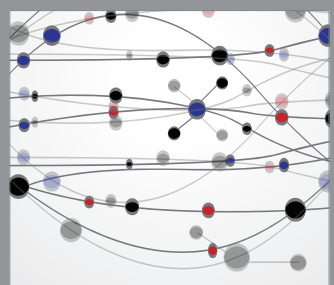

The Scientific World Journal
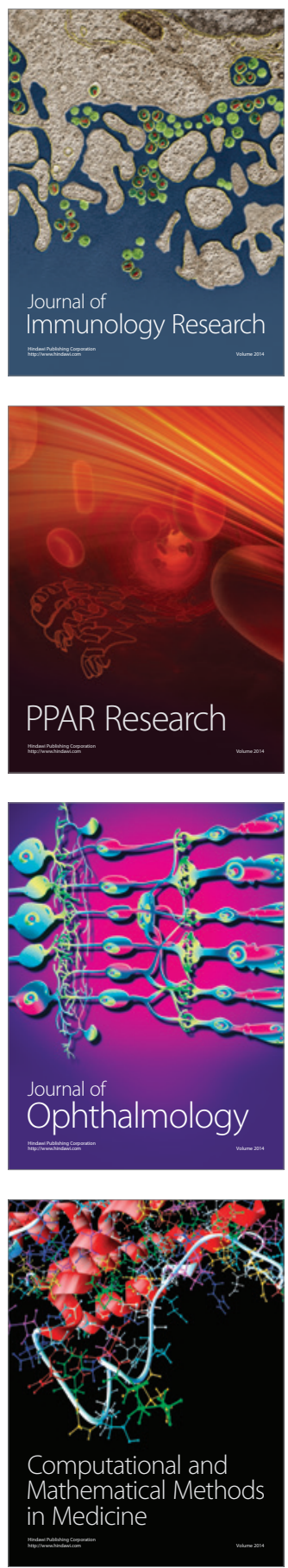

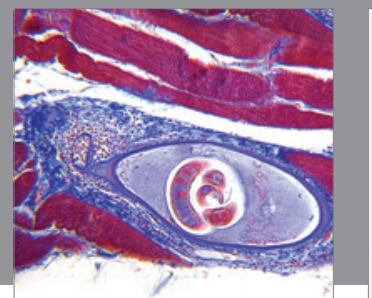

Gastroenterology

Research and Practice
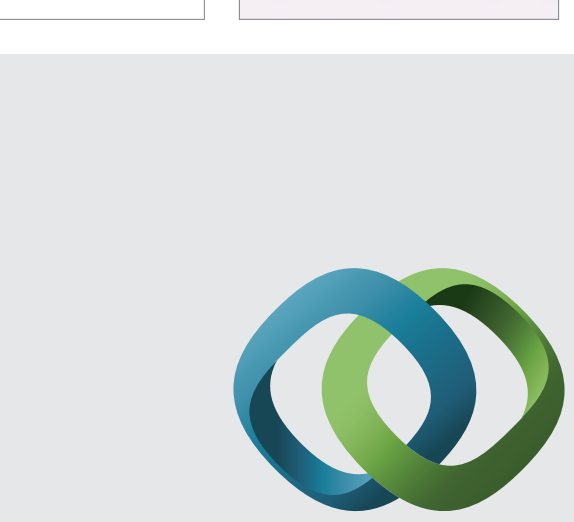

\section{Hindawi}

Submit your manuscripts at

http://www.hindawi.com
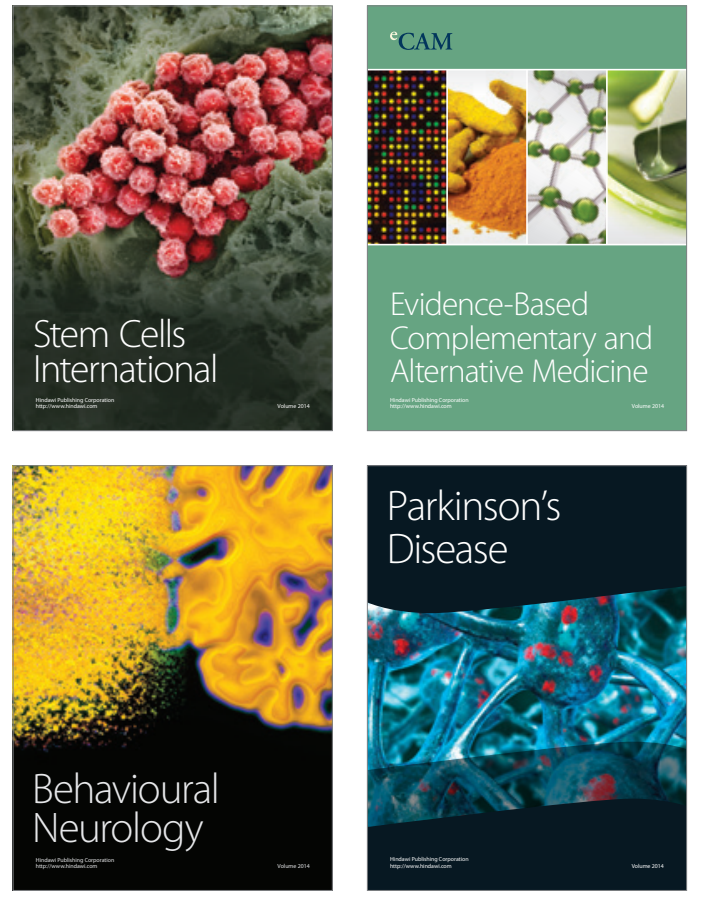
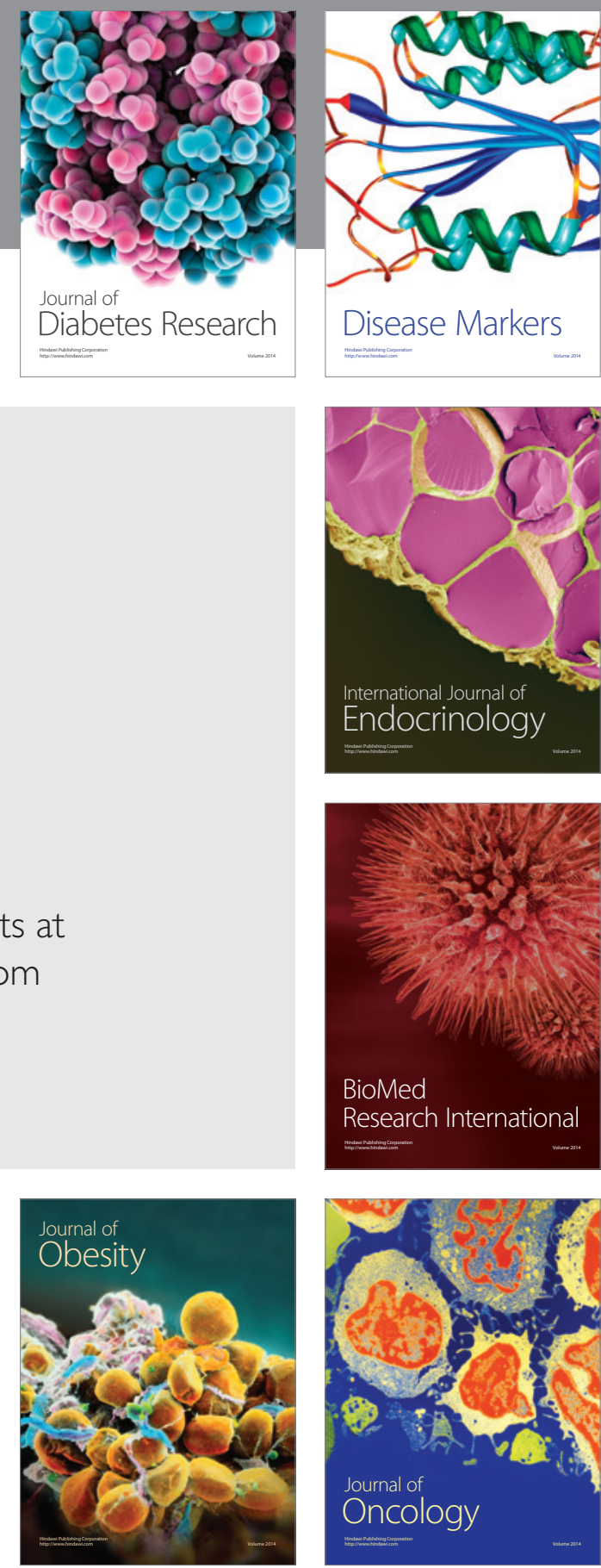

Disease Markers
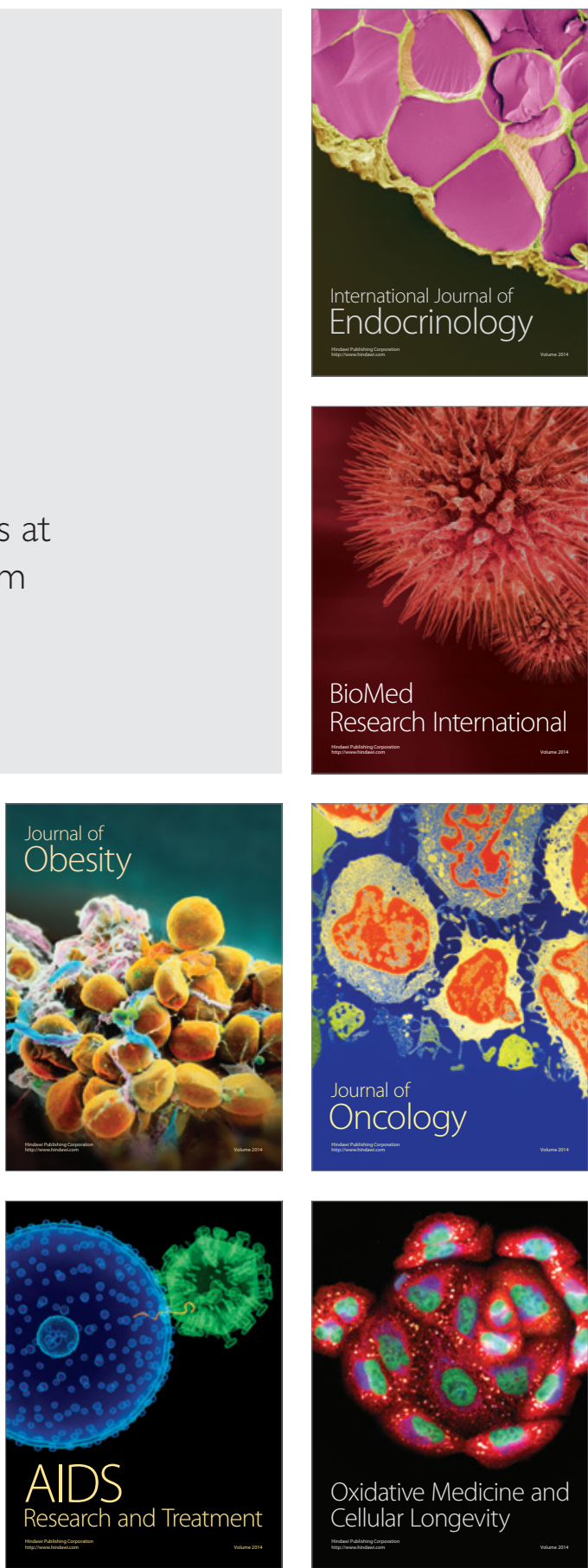